\title{
Norois
}

Environnement, aménagement, société

$244 \mid 2017$

Sport, immigration, urbanisation littorale, climat sahélien, biodiversité

\section{Instruments de l'action publique et approche fixiste de la biodiversité : le cas des inventaires naturalistes}

Tools of public action and fixist approach to biodiversity

Clémence Guimont et Rémy Petitimbert

(2) OpenEdition

Journals

Édition électronique

URL : https://journals.openedition.org/norois/6169

DOI : $10.4000 /$ norois. 6169

ISBN : 978-2-7535-7420-5

ISSN : $1760-8546$

Éditeur

Presses universitaires de Rennes

Édition imprimée

Date de publication : 30 décembre 2017

Pagination : 77-89

ISBN : 978-2-7535-7418-2

ISSN : 0029-182X

Référence électronique

Clémence Guimont et Rémy Petitimbert, « Instruments de l'action publique et approche fixiste de la biodiversité : le cas des inventaires naturalistes », Norois [En ligne], 244 | 2017, mis en ligne le 30

décembre 2019, consulté le 13 janvier 2022. URL : http://journals.openedition.org/norois/6169; DOI https://doi.org/10.4000/norois.6169

(c) Tous droits réservés 


Presses
Universitaires
de Rennes
www.pur-editions.fr

\title{
Instruments de l'action publique et approche fixiste de la biodiversité : le cas des inventaires naturalistes
}

\author{
Tools of Public Action and Fixist Approach to Biodiversity
}

Clémence Guimont*, Rémy Petitimbert

\begin{abstract}
*Auteure correspondante : Tel : 0033676752606
CERAPS, UMR 8026, 1 Place Déliot, BP 629, 59024 Lille Cedex, France (clemence.guimont@hotmail.fr)

(remy.petitimbert@laposte.net)
\end{abstract}

Résumé : La culture du chiffre issue du New public management influence les instruments des experts naturalistes et de l'action publique. Les inventaires naturalistes, en tant qu'instruments territoriaux de l'action publique, entrent en résonance avec l'institutionnalisation du chiffre prescriptif. Ils contribuent à la formation des cadres cognitifs et normatifs de la gestion du vivant, que nous qualifions de fixiste. Ceux-ci tendent à produire une éviction de la perte territoriale de biodiversité au profit d'une gestion par espèces du vivant.

Abstract: New Public Management's numbers- and results-based culture influences the tools used for both public policies and naturerelated assessments. As an instrument of territorial environmental public policy, natural resource inventories are tied to the institutionalization of a prescriptive use of figures. These tools drive the cognitive and normative frameworks used in the management of the living environment towards what we call a "fixist" perspective. As a consequence, they tend to mask biodiversity losses in individual territories in favour of management by living species.

Mots clé : Politiques publiques - indicateurs quantitatifs - inventaire - biodiversité - expertise naturaliste

Keywords: Public policies - quantitative indicators - inventory - biodiversity - naturalistic expertise

\section{INTRODUCTION}

"Ce que nous pouvons préserver, ce n'est pas l'état actuel du monde vivant et de notre planète: c'est sa capacité à renouveler, à évoluer, et à nous permettre d'y vivre. » Jean-Claude Ameisen (2015, p. 62)

La montée en puissance depuis le milieu des années 1980 des logiques néolibérales au sein de l'action publique nationale française a provoqué de nombreuses modifications et ce, tant d'un point de vue cognitif qu'opérationnel (Dardot, Laval, 2009). Une nouvelle ligne théorique de conception de l'État et de son action apparaît. Cette dernière prône la performance à travers la culture du résultat et entraîne, pour Odile Henry et Frédéric Pierru (2012, p. 10), le développement « de nouveaux instruments d'action publique comme la contractualisation et le pilotage par les indicateurs [...]». Désormais, le résultat est au centre de l'attention de 
l'action publique qui importe les outils de gestion et de management de la sphère privée au cœur du pilotage des politiques qu'elle entreprend. Ces derniers, issus du New public management (NPM), ont vocation à amener l'action publique vers la performance en la dotant d'instruments de pilotage à tous les échelons et à toutes les temporalités de la politique menée (Bezès, 2012). La performance résulte d'une mise en compétition des infrastructures et est censée produire une meilleure efficacité dans l'élaboration des décisions et de leurs applications via un recours systématique à l'évaluation des politiques publiques (Perret, 2008). Les principes de la culture du résultat doivent ainsi être traduits dans un langage facilitant comparativité, traçabilité, performativité et évaluation.

Le chiffre apparaît alors dans ce contexte comme un outil fonctionnel pour construire les bases d'un tel langage. Il présenterait l'avantage de décrire objectivement la réalité à appréhender; la richesse nationale, le chômage, ou le nombre d'élèves par classe sont ainsi mesurés par un outil neutre, traduisant d'une manière factuelle et offrant une dimension saisissable en dehors de toute considération subjective (Ogien, 2010). Le chiffre présente aussi un autre avantage : celui de produire une capacité d'injonction, qui correspond à un mode de pensée et un mode d'action qui permettent l'orientation de la planification. Le chiffre est bien plus qu'une modalité de mesure, puisqu'il représente aussi l'objectif à atteindre, le score à dépasser, le résultat qui permet la monstration de la performance de l'action publique. Comme l'explique Robert Salais (2010, p. 135), "le décideur central fixe sans concertation sérieuse (autre qu'interne) un tableau de bord composé d'objectifs quantitatifs à réussir. Tous les acteurs de haut en bas de la filière de la décision publique doivent ensuite s'ingénier pour trouver à leur niveau la manière d'accroître, à moyens constants, la performance telle qu'elle s'inscrira in fine dans le tableau de bord du décideur central. [...] L'enjeu n'est plus d'améliorer directement les situations [...] mais d'obtenir la performance quantitative souhaitée, qu'elle qu'en soit la méthode».

Dès lors, le chiffre n'est plus un instrument de mesure, qui permet d'appréhender une conception de la réalité à des fins d'action, il devient une finalité et est doté d'une dimension performative qui le place comme un score à atteindre (Bruno, Didier,
2013). Auparavant le chiffre venait nourrir une décision politique et il était un outil d'aide à la décision. Les cadres cognitifs et normatifs de la culture du résultat en ont fait un objectif de réussite. Comme l'explique Robert Salais (2010, p. 132) : «Dans le New public management, sous des formes diverses, l'évaluation de la performance quantifiée se trouve internalisée, à la fois comme référence et comme objectif, dans le processus de l'action. » Les données chiffrées expriment alors une certaine réalité du vivant, construite scientifiquement. Elles deviennent alors la principale structure des tableaux de bord de ces politiques, permettant de diagnostiquer la situation initiale (inventaires), de lui donner une orientation (objectifs quantifiés) et d'en évaluer le résultat, définissant ainsi les critères de la performance. Les chiffres deviennent le parangon à travers lequel les sphères publique et scientifique échangent pour déterminer les conduites à tenir. La connaissance offerte par les indicateurs chiffrés n'est donc plus un préalable à l'action permettant de fixer ex ante les modalités de l'intervention publique, elle devient un processus fixant, in itinere, au sein de tableaux de bord, les performances à atteindre pour une action publique efficace.

L'ensemble des politiques publiques sont concernées par cette quantification devenue prescriptive et ce, quels qu soient les champs d'intervention de l'État (la santé [Pierru, 2007], la recherche [Bruno, 2008] mais également la biodiversité [Blandin, 2009]). Selon Florian Charvolin (2003), la particularité des politiques environnementales, aux finalités imprécises et variables ainsi qu'à l'autonomie faible vis-à-vis du politique vont favoriser l'intégration de ces logiques du résultat au sein de ce champ de l'action publique. De leur côté, Valérie Boisvert et Franck-Dominique Vivien (1998) expliquent que la prédominance du chiffre, exprimée notamment à travers la transposition monétaire, dans les politiques de biodiversité proviendrait de sa capacité affichée à gérer les incertitudes inhérentes à la prise de décision. Le chiffre se serait dès lors imposé au sein de «Toutes les catégories d'acteurs en présence, qu'il s'agisse de scientifiques, d'industriels, d'usagers ou d'ONG, [qui] évoquent la perte de richesse que constitue l'érosion de la biodiversité pour justifier sa préservation ou préconiser des principes de gestion soutenable » (Boivert, Vivien, 1998, p. 17). L'intérêt récent de l'action publique pour les 
services écosystémiques est à ce titre révélateur de l'institutionnalisation de la quantification.

Ainsi, l'action publique en matière de biodiversité, influencée par les cadres cognitifs du NPM, se saisit du dispositif chiffre auquel il fait écho. Comme l'explique le ministère de l'Écologie et du Développement Durable, la Stratégie Française pour la Biodiversité (2004, p. 39) doit notamment permettre de «mettre en place un dispositif d'observation opérationnel qui fournisse des données fiables, objectives et représentatives de l'état de la biodiversité, pour le pilotage de la stratégie et de ses plans d'action, ainsi que pour l'information de la société ». L'action publique de biodiversité est ainsi jalonnée depuis son institutionnalisation politique d'instruments de pilotage s'appuyant sur le chiffre pour connaître et évaluer la biodiversité.

Consciente de l'érosion de la biodiversité sur les territoires, l'action publique nationale et locale multiplie, dans cette logique, les recours aux inventaires naturalistes (Arpin, Fortier, Charvolin, 2015). Le vivant fait l'objet d'inventaires destinés à guider l'action publique, qu'il s'agisse de la mise en place des Zones d'intérêts écologiques, faunistiques et floristiques (ZNIEFF) dès 1982 (Mathevet et al., 2010) pour définir les sites écologiques territoriaux remarquables, de l'implication des inventaires pour les sites Nature 2000 (comme le montre Agnès Fortier dans son étude de cas sur le Nord-Pas-deCalais [2009]) ou encore de l'adoption du Système d'information sur la nature et les paysages (SINP) par le ministère de l'Écologie en 2007 (Alphandéry, Fortier, 2015). Selon la méthode employée ${ }^{1}$, ces inventaires recensent et chiffrent les espèces présentes. Il y est ainsi indiqué le nom du taxon, la date, le lieu de l'observation et le nom de l'observateur (Arpin, Fortier, Charvolin, 2015). Pierre Alphandéry et Agnès Fortier soulignent ainsi que la multiplication de ces inventaires commandités par l'action publique donne « une conception de la donnée conçue comme une information objective, définie de manière conventionnelle et facilement mobilisable grâce à des banques de données. Cette seconde conception fait entrer la nature dans le monde des procédures, des normes et de l'abstraction » (Alphandéry, Fortier, 2013, p. 212). Ils

\footnotetext{
1. Pour plus de détails à ce sujet, voir par exemple le cas des inventaires des Zones d'intérêts écologiques, faunistiques et floristiques in Mathevet et al., 2013 .
}

indiquent également que la néolibéralisation de l'action publique tend à accorder une place prépondérante aux instruments de quantification de la biodiversité. Ainsi, depuis les années 1980, les inventaires naturalistes ne sont plus seulement des outils de connaissance scientifique. Ils sont des instruments de l'action publique au sens où ils deviennent des dispositifs prescriptifs pour l'action publique.

Les conditions de construction et d'élaboration de ces inventaires naturalistes ont déjà été longuement étudiées (notamment in Arpin, Fortier, Charvolin, 2015). L'objectif de cet article est de davantage se concentrer sur les effets politiques de l'institutionnalisation de cette mise en chiffres du vivant quant à la gestion territoriale de la biodiversité. Cela passe par l'étude de la place accordée au chiffre produit par la diffusion des inventaires - qui traduit ici les espèces et les individus présents sur un territoire dans la fabrique de l'action publique territoriale en matière de biodiversité. Les politiques territoriales s'inscrivent dans des pratiques expertes qui mobilisent le chiffre comme outil d'élaboration, de gestion et d'évaluation des politiques publiques (Alphandéry, Fortier, 2013). Ainsi, les collectivités territoriales incitent à la production de données sur le vivant, via les politiques d'inventaires naturalistes, qui traduisent la réalité écologique en données vulgarisées pour le décideur. Ces politiques d'inventaires sont généralement de l'ordre de l'organisation de la production de données territoriales sur le vivant (construction de réseaux naturalistes et de plateformes numériques pour recueillir et organiser les données, soutien financier aux associations naturalistes pour le recensement des espèces territoriales, création d'observatoires territoriaux de la biodiversité). Elles sont étroitement liées à celles de gestion de la biodiversité, qui déterminent les sites et espèces remarquables à partir des inventaires menées par les experts locaux, comme les inventaires Zones de protection spéciale (ZPS), Zone naturelle d'intérêt écologique, faunistique et floristique (ZNIEFF), Zone d'importance communautaire pour les oiseaux sauvages (ZICO) ou Natura 2000.

À travers leur utilisation du chiffre, les inventaires représentent une construction rationalisée du savoir et sont un appui à la décision (Arpin, Charvolin, Fortier, 2015; Mathevet et al., 2013). Cette boîte à outils participe de l'orientation et à la légitima- 
tion des choix faits par l'action publique en matière de gestion de la biodiversité. En exprimant par le chiffre des situations relevées par les scientifiques, ces inventaires répondent à une commande de l'action publique qu'ils vont, dès lors, pouvoir guider. Ils représentent en cela des instruments essentiels dans la gestion de la biodiversité car les représentations du vivant, qu'ils incarnent et diffusent, influencent le choix de la gestion par espèces menée par les institutions territoriales.

Les inventaires ont ainsi quatre fonctions principales : 1) délimiter le vivant sur un territoire - qui correspond souvent à une division administrative (communes, régions, départements); le territoire du vivant se construit alors à partir de frontières socialement construites; 2) évaluer, via un travail de quantification, l'état du vivant sur un territoire; 3) traduire la biodiversité, par un travail de formalisation des informations, afin de les rendre appropriables en dehors du seul champ des spécialistes; 4) enfin, permettre un suivi de l'effet des politiques publiques via un processus d'évaluation. Ainsi, « les inventaires représentent plus largement des moyens d'apprentissage, de retour réflexif et d'évaluation de la politique publique. Ils contribuent à réduire le temps entre une décision publique et la mesure de son efficacité » (Arpin, Fortier, Charvolin, 2015, p. 19). Les inventaires naturalistes n'ont donc plus pour seule fonction d'informer.

\section{Cadre théorique}

Étudier ces politiques suppose de s'appuyer sur la sociologie politique, en ce qu'elle permet de rendre compte des conditions de fabrique d'une politique publique (notamment en tenant compte des conditions institutionnelles de son énonciation et de son élaboration). Cependant, il convient de croiser cette approche de science politique avec les apports plus spécifiques de la sociologie environnementale, qui permet d'interroger les conditions de désignation du vivant, de sa prise en considération et de sa gestion. Cela permet de prendre en compte à la fois le contexte institutionnel de création de cette politique de biodiversité (poids des acteurs, contraintes législatives, New public management...) et le contexte scientifique (crise de la biodiversité, expertise scientifique, etc.).
Nous proposons donc l'hypothèse selon laquelle les objectifs en matière de gestion de la biodiversité se sont établis au sein de la «culture du résultat », qui irrigue les politiques publiques. Ces objectifs, plaçant la performance comme finalité de l'action publique, ont alors été traduits par les acteurs de l'action publique au sein d'instruments d'appréhension de la diversité biologique, porteurs des valeurs du management et de la concurrence. L'institutionnalisation du chiffre contribue à faire de l'inventaire naturaliste un instrument de l'action publique (1). Les instruments de l'action publique, loin de répondre à une neutralité axiologique, sont « nourris d'une interprétation du social et de conceptions précises du mode de régulation envisagé » (Lascoumes, Le Galès, 2005, p. 12). Il en est de même pour l'interprétation du vivant. Les inventaires décomposent le vivant en catégories d'espèces, et cela produit des incitations différenciées à l'action, suivant l'intérêt de telle ou telle espèce. La diffusion de ces typologies, permise par un chiffre prescripteur, produit alors une représentation sélective de l'intérêt du vivant, que nous qualifierons ici de fixiste (2). Le chiffre prescripteur fixe ainsi le vivant à un nombre d'espèces et d'individus à un moment $t$, et offre une ligne de fuite de la biodiversité, qui permet de calibrer et d'orienter les politiques publiques. Par conséquent, la diffusion de ces instruments chiffrés contribue à légitimer une vision fixiste du vivant auprès de l'action publique. Cela n'est pas sans conséquences sur les politiques territoriales de biodiversité. Ainsi, c'est une gestion par espèces qui est favorisée (3), répondant aux logiques managériales et gestionnaires du vivant mais qui semble en décalage avec l'érosion territoriale de la biodiversité et aux pressions anthropiques croissantes sur les territoires.

\section{Terrain D'étude ET CADRE MÉTHODOLOGIQUE}

Comment une collectivité territoriale élabore et gère sa politique d'inventaire de la biodiversité? Cette question a fait l'objet d'une étude empirique, pendant deux ans, sur le territoire du Nord-Pas-deCalais. En ayant agi dès le début des années 1990 en matière de biodiversité et étant à l'initiative de nombreux partenariats politiques, la Région NordPas-de-Calais peut être considérée comme un terri- 
toire pilote et moteur dans sa gestion territoriale de la biodiversité. Les travaux d'Agnès Fortier et Pierre Alphandéry sur cette région confortent l'idée que ce territoire est pionnier en matière de gestion de la biodiversité (Alphandéry, Fortier, 2011, 2013, 2015; Alphandéry, Fortier, Sourdril, 2012). Les pollutions liées à l'industrie minière et à l'agriculture intensive ont poussé les acteurs politiques du territoire et les experts régionaux à intervenir en faveur de la biodiversité dès le début des années 1990, notamment en fondant une direction régionale de l'environnement au sein du Conseil régional, en créant l'ancêtre de la Trame verte et bleue et en finançant différentes structures destinées à connaître et à protéger la biodiversité, comme l'Observatoire régional de la biodiversité. L'érosion de la biodiversité sur le territoire, évaluée par l'Observatoire régional de la biodiversité est notamment marquée par la disparition d'une espèce végétale et demie en moyenne tous les ans. Elle préoccupe les acteurs publics, qui agissent dans la perspective de l'endiguer. En cela, le territoire nous semble exemplaire et représentatif des grandes modalités de l'action conjointe de l'action publique et de l'expertise scientifique de la gestion du vivant, bien que nous détaillerons certaines de ses spécificités liées aux acteurs locaux.

La connaissance quantitative au service de la gestion finit par s'imposer au sein de l'action publique, impliquant un recensement systématique de la biodiversité. L'Observatoire de la biodiversité du NordPas-de-Calais, créé en 2010 et financé par les institutions européennes, la DREAL et le Conseil régional, a pour objectif de fournir à l'action publique des indicateurs sur l'état de la biodiversité et des indicateurs sur les conditions de sa gestion politique ${ }^{2}$.

Vingt-neuf entretiens semi-directifs ont été menés avec des membres du réseau de collectes de données naturalistes (2), des administrateurs d'associations naturalistes (3), des responsables des Conservatoires (3), des membres d'associations de protection de l'environnement (3), des administrateurs et des élus des collectivités territoriales (8), de la Direction Régionale de l'Environnement, de l'Aménagement du territoire et du Logement (DREAL) (3) et des organismes publics et chambres consulaires (7). Ces entretiens ont permis d'identifier les méthodes, les instruments et les référentiels des acteurs territoriaux en matière de collecte de données naturalistes et d'en saisir l'influence dans la prise de décision en matière de gestion de la biodiversité territoriale. Cela a été complété par un travail sur les archives de la direction de l'environnement du Conseil régional du Nord-Pas-de-Calais pour analyser les liens entre associations naturalistes et pouvoirs publics (notamment grâce à l'analyse des conditions de contractualisation entre pouvoirs publics et associations naturalistes via la lecture des cahiers des charges, la reconstitution des subventions accordées par le Conseil régional aux associations depuis 1992, etc.). Nous avons pu ainsi étudier les principales politiques locales de biodiversité et leur mise en œuvre sur le territoire depuis 1992 jusqu'en 2015; ce qui correspond à l'époque où les socialistes et les écologistes gouvernaient ensemble la Région. Cette période marque une continuité dans la gestion de la biodiversité territoriale puisque c'est le même personnel politique qui gère la direction de l'environnement du Conseil régional. Bien qu'il y ait eu des évolutions, ces vingt-trois années se caractérisent par l'approfondissement de réseaux naturalistes et de logiques de gestion.

Ce cas d'étude nous permet de saisir la territorialisation de la biodiversité, notamment à travers l'analyse des politiques d'inventaires. Étudier les instruments de recensement et de comptage de la biodiversité à destination de l'action publique (Conseil régional, Direction régionale de l'environnement, de l'aménagement du territoire et du logement, et leurs institutions partenaires) menés par les sociétés savantes et les associations de protection de l'environnement dans la région Nord-Pas-de-Calais, permet ainsi d'interroger les conditions d'élaboration (protocoles, conditions de l'aide publique, cadrage de la commande publique, etc.), de validation scientifique (évaluations par les pairs, valorisation scientifique...), et de transmission des informations naturalistes (modalités de leurs publicisations, de leurs présentations et de leurs diffusions) pour en mesurer les effets sur la gestion de la biodiversité.

2. Notamment en analysant les montants financiers accordés par chaque institution régionale à la biodiversité, voir le site de l'Observatoire de la biodiversité dans le Nord-Pas-de-Calais : [http://www.observatoire-biodiversite-npdc.fr/fiches.html] (consulté le 19 mai 2016). 


\section{LES CONSÉQUENCES DE L'INSTITU- TIONNALISATION DU CHIFFRE : L'INVENTAIRE COMME INSTRUMENT DE L'ACTION PUBLIQUE}

Les instruments utilisés pour constituer et diffuser les inventaires sont représentatifs à la fois de cette culture du chiffre et de sa capacité présupposée à objectiver le savoir. Bien qu'ils ne puissent être jamais exhaustifs, les inventaires naturalistes ont pour vocation de recenser les espèces présentes sur un territoire donné et le nombre d'individus qui s'y trouvent. À titre d'exemple, les fiches-indicateurs d'état proposées par l'Observatoire régional de la biodiversité dénombrent le nombre d'espèces patrimoniales et rares présentes sur le territoire ${ }^{3}$. Ils décrivent ainsi notamment la nature à partir d'une vision comptable. Le vivant y est en effet traduit par les chiffres. S'intéresser à la phase de diffusion de l'inventaire, en tant que producteur de chiffre à des fins de représentation de la diversité du vivant permet alors de dépasser une critique de la méthode d'élaboration de cet instrument d'action publique pour nous intéresser à ses effets en termes d'action publique. Il s'agit alors de rendre compte non pas de la méthode de production de l'inventaire mais des effets qu'il induit sur l'action publique en tant que produit fini. En fin de compte, l'espèce est objectivée dès qu'elle devient un chiffre, qui lui confère une nouvelle identité, réduite à quelques caractéristiques quantifiables. Ce chiffrage contribue néanmoins à réduire la matière vivante en unités décomposables, qui permet d'élaborer une comptabilité. Cette simplification de la matière vivante permet de décrire objectivement le milieu naturel - ou tout au moins en unité objective -, sans rentrer dans des controverses scientifiques sur les conditions d'évolution des espèces. Mais une telle transformation du vivant est bien souvent une simplification du rapport au vivant; la donnée ainsi produite est détachée des conditions écologiques de sa production (l'inventaire d'une espèce n'indique pas directement l'état écologique de l'écosystème dans laquelle elle évolue). Ce travail d'objectivation, procédant à une réduction de la réalité à des fins de perception, a ainsi tendance à isoler artificiellement la donnée vivante des

3. Voir la page internet de l'Observatoire régional de la biodiversité en NordPas-de-Calais : [http://www.observatoire-biodiversite-npdc.fr/indicateurs. html], consultée le 29 septembre 2017. conditions sociales et politiques de sa production. Le chiffre produit ainsi à la fois une artificialisation du vivant ainsi qu'une forme d'objectivisation de sa présentation, et à terme, une certaine représentation du vivant (Arpin, Charvolin, Fortier, 2015).

De plus, la production des données produites devient une finalité en soi : la recherche de la meilleure technique de production de données, ainsi que les conditions d'une meilleure utilisation de ces données (diffusions scientifiques, création de bases de données, etc.) deviennent des priorités dans l'inventaire. Le chiffre présente la propriété, essentielle dans le monde scientifique, de pouvoir apporter une forme de traçabilité du travail mené. En portant le regard sur la manière dont le chiffre a été produit, toute personne intéressée par le résultat et souhaitant se référer à la manière dont il a été obtenu pourra se pencher sur les modalités d'évaluation, de quantification, sur la manière dont les variables sont construites (le terrain d'enquête, la population suivie, les temporalités d'observation...) et les partis-pris de la recherche, les cadres théoriques sousjacents, etc. Ainsi, les modalités d'obtention du chiffre sont appréhendables, même si elles peuvent être techniques, et facilitent une double traçabilité méthodologique et théorique.

Le chiffre dispose également d'une autre propriété mobilisée au sein de l'action publique : l'établissement d'objectifs d'efficacité et d'efficience ${ }^{4}$. Ainsi, qu'ils soient propres au champ de la recherche (nombres de d'actes scientifiques...) dans un contexte où les effets de concurrence sont développés (via des financements d'appels à projets... cf. Bruno, 2008) ou qu'ils concernent l'efficience des préconisations, les objectifs sont traduits en termes chiffrés, qu'ils soient alors exprimés en termes monétaires, d'espèces ou encore de publications. Le chiffre est alors une modalité de management qui apparaît comme équitable car il soumet chacun à l'évaluation via des critères de quantification et d'observation eux-mêmes souvent chiffrés et bénéficiant à la fois d'une valeur sociale et d'une légitimité.

Les inventaires sont l'incarnation de la culture du chiffre. Ils comptabilisent les populations d'espèces et permettent de mieux connaître les milieux via le recensement de l'ensemble des espèces et

\footnotetext{
4. L'efficacité désigne la mise en relation des résultats par rapport aux objectifs que se fixe l'action publique. L'efficience désigne la mise en relation des résultats par rapport aux moyens qui ont été accordés.
} 
des individus présents. Les inventaires naturalistes répondent ainsi à une exigence de l'action publique. La capacité de mettre en chiffre le vivant est une commande des pouvoirs publics, qui agissent dans ce cadre gestionnaire. Un enquêté, président d'une société savante ${ }^{5}$, explique ainsi la priorité que s'est fixée sa société : "Trouver les outils [ndlr : les outils pour chiffrer les différentes espèces de champignons sur le territoire régional] pour arriver à apporter des réponses aux gestionnaires, compatibles à leur attente dans un délai raisonnable par rapport à ce qu'ils attendaient comme élément. » Il illustre la pression plus ou moins implicite des pouvoirs publics pour que le scientifique soit en mesure de leur fournir les chiffres leur permettant de déterminer leur action. L'institutionnalisation de cette procédure scientifique au sein des arènes publiques fait que les pouvoirs publics exigent une harmonisation des procédures et des règles d'élaboration de ces inventaires, afin qu'ils soient tous établis à partir des mêmes méthodes (Alphandéry, Fortier, 2013). La marge d'autonomie des collecteurs de données se réduit tandis que la place des inventaires dans les dispositifs de l'action publique s'accroît. Les inventaires naturalistes deviennent indispensables pour l'action publique territoriale, qui s'appuie sur ces données pour décider des espèces et des espaces à protéger et des orientations en matière de gestion de la biodiversité. Ils sont ainsi l'instrument par lequel l'action publique s'approprie les problèmes liés à la biodiversité car ils sont le relais entre les experts et les acteurs publics.

Le chiffre tend ainsi à se substituer à la réalité même de la situation décrite. En étant alors appréhendé par le chiffre, réduit à une agrégation de données, le vivant n'est plus représenté dans toute sa complexité et les liens entre les espèces et les écosystèmes dans lesquels elles se trouvent ne peuvent pas être traduits en chiffres. Il s'ensuit alors une nécessaire réduction de la réalité du vivant. Or il apparaît que réduire le vivant aux seules variables appréhendables par le chiffre participe d'une minimisation non seulement de ses composantes, de son fonctionnement et des interactions mais à travers eux, à une minimisation de l'érosion de la biodiversité.

Les inventaires ne sont pas des instruments neutres de l'action publique. Arpin, Charvolin et

5. Entretien du 7 mai 2015.
Fortier (2015, p. 17) indiquent que les inventaires sont devenus un lieu de pouvoir avec la montée du néolibéralisme, en ce sens qu'ils constituent un « dispositif ayant pour effet majeur l'attribution d'un sens au réel et sa mise en visibilité ». Ils produisent une mise en ordre du vivant en le décomposant en catégories et en individus. Leur diffusion donne ainsi une certaine image du vivant - que nous définirons et qualifierons de «fixiste »-auprès des acteurs publics régionaux et locaux. Elle influence les politiques territoriales de biodiversité et produit une tendance à la minimisation de la perte globale de biodiversité en favorisant une gestion chiffrée par espèces qui met l'accent sur les espèces rares plutôt que sur les tendances générales de l'érosion du vivant.

\section{LA CULTURE DU CHIFFRE PRESCRIPTEUR, PRODUCTRICE D'UNE APPROCHE FIXISTE DU VIVANT}

La Région Nord-Pas-de-Calais inclut une grande diversité d'acteurs experts (savants et profanes avec les conservatoires, les associations savantes, les associations de protection de l'environnement, etc.) menant des inventaires naturalistes - qu'ils s'agissent des inventaires Zones de protection spéciale (ZPS), Zones Naturelles d'Intérêt Faunistique et Floristique (ZNIEFF), Zone d'importance communautaire pour les oiseaux sauvages (ZICO), Natura 2000, etc. ou des inventaires menés pour les rapports et les études de l'Observatoire de la biodiversité du Nord-Pas-de-Calais - pour la gestion de la biodiversité territoriale. Dans la région Nord-Pas-de-Calais, le Conservatoire botanique de Bailleul sert d'espace de convergence de nombreux acteurs de la biodiversité et est souvent mobilisé par les acteurs politiques locaux pour organiser les politiques de biodiversité. Il se trouve, par exemple, au cœur du Réseau des acteurs de l'information naturaliste (RAIN). De son côté, le Groupe ornithologique du Nord ou la Société mycologique du Nord (Alphandéry, Fortier, 2013) fondent leur légitimité dans leur capacité à produire des données à partir du travail des inventaires.

La prédominance de la phytosociologie, liée à la renommée internationale du Conservatoire botanique de Bailleul - promoteur de cette discipline explique en partie la place essentielle qu'occupe 
l'inventaire dans la production de la connaissance de la biodiversité régionale ${ }^{6}$. Ces acteurs produisent ensemble des inventaires naturalistes à partir d'une même technique, qui est celle de la classification par espèces. Les méthodes de connaissance de la biodiversité territoriale s’inscrivent dans la culture du résultat et de la performance car les chiffres fournis par les inventaires représentent plus qu'un outil d'aide à la décision : ils contribuent à renforcer une approche managériale de la biodiversité.

Ces acteurs développent ainsi des méthodes de recensement de la biodiversité commune, d'autant plus que le manque de moyens humains, financiers et matériels pour la production de ces inventaires incite les acteurs naturalistes à travailler ensemble pour produire et organiser des données sur l'état de la biodiversité territoriale. Ces difficultés organisationnelles liées au peu de moyens dont ils disposent ont une conséquence majeure. Elles les obligent à hiérarchiser les lieux et les espèces à étudier prioritairement. Ainsi, comme nous l'explique une chargée de mission du Conseil régional en détaillant les rapports naturalistes que le Conseil régional reçoit ${ }^{7}$, les inventaires sur les réservoirs de biodiversité, riches en espèces rares et menacées sont favorisés, au détriment de la biodiversité ordinaire, ce qui pèse inévitablement sur la connaissance du vivant. Le vivant étant décrit à partir de la faisabilité organisationnelle pesant sur les experts, cette sélectivité liée à des contingences matérielles renforce une diffusion partielle de l'état du vivant ${ }^{8}$.

Ces acteurs produisent ensemble des inventaires naturalistes qui sont, comme le définit Gouyon $(2013)^{9}$ « une production de systèmes ordonnant le vivant ». La prédominance des acteurs précédemment cités et la présence de nombreux naturalistes confirmés ou amateurs dans les associations de protection de l'environnement chargées de réaliser ou de participer à ces inventaires valorisent la classification par espèces. Cette dernière met en ordre le

6. Entretien du 28 avril 2015 avec un chargé de mission du Conservatoire botanique de Bailleul.

7. Entretien du 23 juin 2015 avec une chargée de mission du Conseil régional.

8. Entretiens des 28 avril 2015, 7 mai 2015 et 8 juin 2015 avec des membres de Conservatoires et de sociétés savantes productrices de ces inventaires.

9. Conférence, Université de Lausanne disponible sur [https://www.unil. $\mathrm{ch} / \mathrm{gse} / \mathrm{fr} / \mathrm{home} / \mathrm{menuinst/la-faculte/seminaire-interfacultaire/archives/}$ la-biodiversite/programme-2013/pierre-henri-gouyon.html] (consulté le 27 avril 2016). vivant en valorisant la biodiversité spécifique plutôt que la biodiversité génétique ou écosystémique.

Toutefois Alain Prochiantz explique que bien que les mathématiques soient un outil de travail, leurs résultats ne doivent pas devenir « une représentation qui se donnerait pour l'essence du vivant quand elle ne peut en être qu'une simplification » (Prochiantz, 2012, p. 110). Le travail des inventaires, via la mise en chiffres, ne peut être une restitution de la complexité du vivant. Ainsi, la diffusion des inventaires et leur médiatisation, etc. participent à construire une vision partielle de la biodiversité, que nous qualifions ici de fixiste, c'est-à-dire qui se définit par une représentation du vivant comme un ensemble d'espèces stables dans le temps.

Tout d'abord, le recensement des espèces par le chiffre transmet à l'action publique une approche qui segmente le vivant en unité individuelle, où l'unité - l'espèce - est étudiée comme segment autonome. Cet ensemble disjoint d'espèces recensé dans le temps renforce l'idée qu'il y aurait une continuité de l'espèce malgré le fait que celle-ci peut évoluer.

De plus, cette autonomie de l'espèce traduite au travers du chiffre minimise les interactions écosystémiques. L'espèce devient un chiffre, isolé de ses conditions écologiques de production et qui n'est plus vraiment représentatif du milieu écologique dans lequel il s'inscrit (Alphandéry, Fortier, 2015). Les variations du vivant, les connexions entre les espèces et les milieux et les interdépendances entre les espèces n'apparaissent pas dans cette traduction mathématique qui, en conséquence, les invisibilise au sein des inventaires à disposition de l'action publique. Ainsi, les êtres vivants sont présentés et organisés par le biais d'unités indépendantes, et de ce fait les instruments de recensement par espèces ne sont pas en mesure de faire apparaître la complexité des liens au sein d'un écosystème. Et pourtant, et nous suivons en cela Casetta (2014, p. 140) : "Les espèces sont trivialement les unités de base de la diversité spécifique, laquelle est un indicateur parmi d'autres de la biodiversité en général (un indicateur se rapportant à une ou plusieurs caractéristiques covariant avec la propriété complexe correspondante). » Les espèces sont ainsi le principal élément recensé, réduisant en quelque sorte la complexité du vivant - qui n'est pas toujours saisissable par le chiffre - à la somme des diverses espèces, saisissable par tous les acteurs. 
Ces inventaires, en devenant le cadre cognitif de l'action publique territoriale, diffusent également l'idée d'un ensemble stable d'espèces dans le temps. Les données chiffrées, qui figent l'état des espèces au moment de leur observation par les collecteurs de données, se substituent à l'évolution du vivant. Les inventaires énoncent l'état d'une espèce à partir de sa présence au moment de son observation scientifique, qui appartient déjà au passé. En effet, l’inventaire rend compte d'une situation existante au moment du dénombrement, qui indique la situation de vie passée de l'espèce. Il élabore et diffuse en conséquence une vision fixiste du vivant, car elle clôt la représentation de l'espèce en fonction de son état passé et ne prend pas en compte la capacité d'évolution, de mutation continue d'une espèce, résultant des interactions avec un écosystème instable et évolutif.

Par conséquent, ces inventaires chiffrés, référentiel de l'action publique, font obstacle à une gestion évolutionniste. La diffusion des inventaires tend à insister sur la préservation d'écosystèmes quantifiés et bien identifiés. Rappelons pourtant que la théorie darwinienne conçoit le vivant comme instable et ne l'envisage pas comme un état mais comme un processus (Darwin, 1862). Le vivant est un ensemble en devenir, en expansion évolutive et imprédictible, car guidé produit par le hasard. Comme le rappelle Céline Granjou : «Dans la théorie de l'évolution, la vie en effet est définie [...] en termes de son historicité, de son ouverture aux événements et finalement de son interdétermination » (Granjou, 2015, p. 47). Ses évolutions ne peuvent être déterminées à l'avance car elles se produisent selon les aléas (conditions de l'adaptation aux évolutions de l'environnement, possibilités de coopérations entre espèces, etc.) et les hasards du futur. « Les espèces changeant au cours du temps (ce qu'on appelle leur évolution), leur ensemble de propriétés (c'est-à-dire leur essence) varie au cours du temps. Appliquer le concept de sorte naturelle ainsi compris aux espèces serait dénier leur variation et leur caractère historique »(Hoquet, 2014, p. 316). Le vivant est ce qui est tourné vers un futur interdéterminé, ce qui inclut l'évolution potentielle des espèces mais il n’est « pas contenu ni dans le passé ni dans le présent » (Granjou, 2015, p. 48).

Or, ces inventaires, malgré leurs limites liées à leurs difficiles conditions financières, humaines et temporelles de production, sont reconnus comme légitimes par les autorités locales. Ils représentent en effet une approche chiffrée valorisée par l'action publique. Comme nous l'indique un enquêté, président d'une société savante, à propos du Conseil régional : "Comme on a développé des indicateurs et des outils d'analyse des inventaires, à partir du moment où l'on met en ouvre cette analyse, le gestionnaire a quelque chose qui est interprétable et qui donne des indications sur ce qu'il doit faire comme mesures de gestion $^{10}$. » L'inventaire devient un outil et un référentiel pour l'action publique (Arpin, Charvolin, Fortier, 2015). C'est donc à partir de ces instruments de recensement que les parcs naturels régionaux, les Conseils régionaux et généraux et la DREAL mettent en œuvre leurs programmes et leurs politiques publiques. Par exemple, la liste définie par arrêté préfectoral des espèces protégées en région Nord-Pas-de-Calais s'appuie sur ces inventaires. Cette liste joue un rôle déterminant dans l'autorisation de construction d'infrastructures et dans les dossiers à instruire pour demander des dérogations sur les espèces protégées. La conséquence de la diffusion de cette approche chiffrée - prescriptive pour l'action publique - est qu'elle contribue à légitimer une perspective fixiste et influence le choix de la gestion par espèces.

\section{L'APPROCHE FIXISTE ET LA GESTION PAR ESPÈCES MENACÉES}

Le classement selon la rareté ou l'abondance des espèces entraîne une forme de hiérarchisation du vivant. La méthodologie de l'Union internationale pour la conservation de la nature (IUCN) recommande d'établir l'indice de menace et l'indice de rareté des espèces à partir de quatre critères : l'effectif, l'aire de distribution de l'espèce, sa démographie et sa probabilité d'extinction estimée à l'aide de modèles (Couvet, Vandevelde, 2014). Les espèces sont ainsi catégorisées entre celles qui sont communes, celles qui sont menacées ou celles qui sont en voie de disparition. De fait, les inventaires régionaux s'appuient sur cette méthode. Comme le dit un enquêté, membre du Conservatoire botanique de Bailleul - non sans humour ${ }^{11}$ - : «On applique

10. Entretien du 7 mai 2015 avec un ancien président d'une société savante du Nord-Pas-de-Calais.

11. Entretien du 28 avril 2015 avec un membre du Conservatoire botanique de Bailleul. 
la méthodologie à l'ensemble de la flore, ça permet de dresser ce genre de trucs imbuvables qu'on appelle les catalogues floristiques avec l'ensemble des espèces, leur statut d'indigénat, la rareté, le niveau de menaces et l'explication et à partir de là on définit aussi des listes d'espèces patrimoniales, on donne des seuils de rareté à partir desquelles on définit les espèces comme patrimoniales, on donne des indications sur les espèces protégées. » Ces inventaires représentent la base de données principale sur laquelle s'appuient les experts chargés de dresser les listes rouges des espèces menacées. L'Observatoire régional de la biodiversité établit ainsi des listes d'espèces locales à protéger à partir des informations chiffrées produites par le travail d'inventaire.

Les instruments de comptabilité de la biodiversité tendent à induire un raisonnement par espèces, donnant une perspective abstraite au vivant, lui conférant ainsi une dimension fixiste. L'action publique, par son souci de s'appuyer sur des données objectivables (et il faut aussi le souligner sur la base de son incompétence technique - les collectivités ne disposant bien souvent pas de services internes leur permettant de procéder à de telles évaluations, comme c'est le cas au sein des Conseils départementaux du Nord et du Pas-de-Calais et au sein du Conseil régional du Nord-Pas-de-Calais) vont favoriser la production de cette perspective fixiste, au détriment des autres caractéristiques du vivant, notamment son potentiel évolutif. D’autres dispositifs, comme la Trame verte et bleue (TVB) s'inscrivent dans une telle politique. La TVB valorise la circulation des espèces identifiées, par la formation de corridors écologiques. Mais c'est une approche par milieux et par habitats écologiques qui est favorisée puisque l'objectif est bien de maintenir des connexions entre les différents écosystèmes pour assurer la résilience des espèces. Cependant, les instruments proposés dans la région Nord-Pas-de-Calais pour tracer la cartographie de ces corridors écologiques restent associés à une approche fixiste du vivant puisqu'ils dessinent le territoire en fonction des corridors écologiques actuels, sans être en mesure d'évaluer les évolutions de ceux-ci, et notamment d'anticiper les bouleversements liés au changement climatique ${ }^{12}$. Ainsi, ils ont tendance à figer la biodiversité sur le territoire à partir de son état actuel. En outre, ces

12. Entretien du 16 octobre 2015 avec une chargée de mission du Conseil régional du Nord-Pas-de-Calais. corridors écologiques sont proposés à partir d'une étude des espèces menacées sur le territoire, établie à partir des inventaires naturalistes. La place qu'occupe l'inventaire naturaliste en tant qu'instrument de l'action publique reste ainsi majeure. L'évaluation de la Charte du parc naturel régional du Scarpe-Escaut illustre par exemple le poids du raisonnement par espèces et notamment la prédominance des espèces dans l'évaluation des dispositifs de gestion ${ }^{13}$. Pour évaluer l'amélioration des habitats écologiques, des fiches-indicateurs par espèces ont été conçues, qui devaient révéler le bon état écologique de la biodiversité. Comme nous l'a indiqué un enquêté ${ }^{14}$, salarié dans le parc naturel régional du Scarpe-Escaut, ces fiches par espèces peuvent donner des résultats lacunaires ou erronés. Par exemple, la disparition des hirondelles de fenêtre n'est pas nécessairement un indicateur du mauvais état écologique du parc, car les hirondelles ont pu avoir une migration compliquée liée à de mauvaises conditions climatiques. Toutefois, l'habitus professionnel et la nécessité de constituer des bases de données cumulées pour rassurer l'ensemble des autres acteurs publics comme les municipalités ou le Conseil régional obligent à une production chiffrée. Notre enquêté a ainsi souligné les difficultés pour sortir de ce raisonnement et de cette évaluation par espèces, en indiquant que les parcs naturels régionaux étaient toujours à la recherche d'autres méthodes d'évaluation sans être encore parvenus à produire du chiffre au-delà du recensement d'espèces ${ }^{15}$. Le fait que l'Observatoire régional de la biodiversité n'ait pas encore conçu d'autres inventaires qui permettent de penser le vivant dans sa complexité et dans sa dynamique démontre les difficultés à sortir du raisonnement par espèces.

Ce raisonnement par espèces induit une gestion et une préservation de la biodiversité par les acteurs publics centrées sur les espèces menacées ou qualifiées d'emblématiques. Le manque de moyens

13. La charte peut être consultée sur [http://www.pnr-scarpe-escaut.fr/ contenu-standard/que-dit-la-charte-du-parc-2010-2022] (consulté le 27 avril 2016).

14. Entretien du 14 octobre 2015 avec un chargé de mission du Parc naturel régional du Scarpe-Escaut.

15. Il nous semble qu'il y ait un décalage entre les sciences de la conservation et les outils mis à disposition par l'expertise scientifique concernant une approche évolutive des politiques de biodiversité. Pour plus de détails sur ce sujet : Robert A. et al., "Fixism and conservation science », Conservation biology, $\mathrm{n}^{\circ}$ 4, vol. 31, p. 781-788; Sarrazin F., Lecomte J., 2016, «Evolution in the Anthropocene », Science, n 351, vol. 6276, p. $922-923$. 
humains, financiers et matériels, allié à la hiérarchisation du vivant par la liste rouge des espèces menacées incitent les acteurs publics à centrer leurs efforts sur quelques espèces. Un enquêté établit ainsi le lien entre les travaux d'inventaires naturalistes et les choix en matière de gestion de la biodiversité : "On va travailler, pas sur toutes les espèces, avec cette hiérarchisation de menaces et de rareté et on va cibler des actions de conservation sur les plus menacées ${ }^{16}$. " Imprégnés de la culture du chiffre et soucieux de pouvoir évaluer les effets de leurs politiques, les acteurs publics comme la DREAL - via les arrêtés préfectoraux d'espèces protégées ou les plans de maintien de certaines espèces comme le phragmite aquatique - ou le Conseil régional consacrent de grands moyens financiers et politiques vers la préservation de ces espèces menacées. En 2012, la DREAL consacre $10 \%$ de son budget biodiversité à la préservation des espèces, sans compter les subventions accordées aux Conservatoires naturalistes, aux réserves naturelles, etc. En 2010, le Conseil régional Nord-Pas-de-Calais accorde 462638 d'euros uniquement à la réintroduction et à la restauration de certaines espèces ${ }^{17}$. Ainsi, le Conseil régional NordPas-de-Calais développe entre 2010 et 2015 une politique visant à réintroduire la loutre et le castor, deux espèces dont le potentiel de sympathie est élevé dans l'imaginaire collectif. L'action politique est donc à la fois pédagogique - à travers le choix de certaines espèces elle espère ainsi une sensibilisation accrue envers la biodiversité - et médiatique car il est aisé de communiquer sur ce type d'espèces. Cette approche sélective renforce ainsi la singularisation de l'espèce particulière comme moteur principal de fonctionnement d'un écosystème, ce qui réduit fortement sa complexité. Un enquêté argumente ainsi le choix de l'action publique :

"Alors il s'agit pas de réintroduire n'importe quelle espèce qui n'a rien à faire là, mais une espèce qui avait quelque chose à faire ici, si possible à poil, si possible sympathique, un peu peluche, ça aurait un intérêt. Donc on a listé un certain nombre d'espèces et voilà. [...] Oui, allons-y, listons toutes les espèces, mais mettons au fronton pas un insecte, pas un reptile, il faut mettre quelque chose qui donne envie,

16. Entretien du 28 avril 2015 avec un membre du Conservatoire botanique de Bailleul.

17. Voir les chiffres fournis par l'Observatoire de la biodiversité : [http://www. observatoire-biodiversite-npdc.fr/fiches.html] (consulté le 17 mai 2016). c'est pas histoire d'être faux-cul mais faut arrêter de se tirer des balles dans le pied. Qui plus est, des espèces comme la loutre et le castor sont des espèces ingénieurs, donc ils vont faire le boulot Trame verte et bleue à notre place, aux endroits où ils seront ${ }^{18}$. "

Cet entretien, expressif, illustre les tensions résultant des choix politiques effectués par les acteurs publics comme les collectivités territoriales ou la DREAL, qui se font en grande partie à partir d'espèces protégées. Les acteurs institutionnels semblent privilégier la visibilité d'une certaine forme de biodiversité, qui allie à la fois l'incarnation de cette biodiversité (l'espèce singulière) et la réalisation d'une politique publique (avec le souci de sa mise en scène). Il ne s'agit plus d'interroger la dynamique d'ensemble du vivant et ses potentielles évolutions, ses capacités de résilience face aux perturbations climatiques : l'action publique concentre ses moyens financiers et humains sur des espèces qui pourront le plus facilement répondre aux objectifs de valorisation d'une politique.

En se centrant sur des espèces à forte portée symbolique mais dont la disparition du territoire illustre leurs difficultés à s'adapter au sein des écosystèmes contemporains, ces dispositifs de réintroduction d'espèces minimisent l'évolution de l'environnement de vie de ces espèces et de certains déjà disparues; elles contribuent alors à maintenir artificiellement certaines espèces (investissement dans le maintien ou la réintroduction), alors que celles-ci n'ont plus toutes les conditions réunies pour s'adapter face à un espace de vie en évolution. Des écologues ont montré le poids minimum que représentaient ces espèces menacées sur la dynamique du vivant (Couvet, Vandevelde, 2014). Ils indiquent que les espèces menacées représentent un faible pourcentage des espèces d'un écosystème. Leur rareté et leurs faibles effectifs font qu'ils ont un faible potentiel de diversité génétique et qu'ils sont peu susceptibles de conduire à l'apparition de nouvelles espèces ou de nouvelles adaptations. Ils concluent ainsi que « le potentiel évolutif associé aux espèces menacées serait donc faible» (Couvet, Vandevelde, 2014, p. 194).

En s'appuyant sur cette culture du chiffre, ces politiques publiques développent une gestion centrée sur le maintien d'espèces, au détriment, parfois, de la valorisation d'une dynamique du vivant

18. Entretien du $1^{\text {er }}$ juin 2015 avec un élu du Conseil régional. 
et de son évolution hasardeuse. L'action publique s'éloigne ainsi de la dynamique systémique et évolutive du vivant en privilégiant une approche comptable, plus rassurante car plus facilement évaluable et appropriable par les pouvoirs publics. Une telle politique permet aussi de rendre plus compatible la gestion de la biodiversité avec les autres politiques territoriales (notamment les enjeux agricoles - avec la gestion des espèces envahissantes, le contrôle des « ravageurs »-, la gestion des politiques de chasse et le contrôle des prédateurs -, etc.)

Mais la prise en compte de la biodiversité ordinaire représente un vrai défi pour l'action publique et « suppose une plus grande attention aux dynamiques du vivant de la part de l'ensemble des acteurs sociaux dans la totalité de leurs actions » (Couvet, Vandevelde, 2014, p. 199). De telles politiques publiques contribuent à promouvoir une vision interventionniste, organisatrice et planificatrice des pouvoirs publics dans la gestion du vivant. Cela laisse supposer qu'ils pourraient ainsi, grâce à la connaissance scientifique, maintenir une permanence de la biodiversité, compatible avec les priorités de l'usage des territoires (agriculture, aménagement urbain, mobilité...); l'état antérieur évalué pourrait ainsi être maintenu, moyennant quelques aménagements ou compensation, de manière permanente. Ainsi, l'action publique focalise ses moyens et ses politiques sur quelques pans spécifiques de la biodiversité, notamment mis en valeur par cette culture du chiffre, sans posséder les instruments et les connaissances pour saisir l'approche systémique et les dynamiques globales du vivant, dont notamment son érosion.

Malgré cela, les indicateurs fournis par l'Observatoire régional de la biodiversité indiquent les difficultés actuelles des pouvoirs publics à endiguer la perte globale de biodiversité sur le territoire du Nord-Pas-de-Calais : la perte d'espèces, qu'elles soient communes ou rares est clairement évaluée. D'après l'Observatoire régional de la biodiversité, en 2015, 56 \% des espèces d'oiseaux du Nord-Pasde-Calais, autrefois communes, sont aujourd'hui classées comme "oiseaux rares » et il en est de même pour $45 \%$ des espèces végétales présentes en région ${ }^{19}$. Ceci signifie que les inventaires par espèces et la gestion par espèces ne sont pas actuel-

19. [http://www.observatoire-biodiversite-npdc.fr/fichiers/documents/bilannature-orb-2015.pdf] (consulté le 17 mai 2016). lement en mesure de fournir les réponses à l'érosion du vivant. L'insuffisance des réponses proposées par l'action publique locale est liée au fait qu'elle n'est pas à même de concevoir la complexité et la dynamique du vivant car elle ne perçoit le problème que selon une vision partielle. Cette vision est réductrice à la fois parce que le chiffre sur un territoire ne saurait donner l'idée de la situation plus globale que représente la perte de biodiversité et parce que le chiffre ne permet d'appréhender qu'une dimension de la complexité du vivant. La perception de ce problème induit des instruments de gestion qui ne parviennent pas à endiguer la perte territoriale du vivant.

Mais l'érosion du vivant complique la culture du chiffre. Les méthodes scientifiques comme les cultures politiques des décideurs peinent à appréhender la perte globale du vivant. Si le chiffre permet de comprendre certains pans de l'évolution actuelle du vivant, il ne saurait permettre de mesurer l'ensemble des interactions en jeu dans l'évolution du vivant. Le chiffre ne peut saisir les conséquences de la disparition de certaines espèces et la transformation que cela induit dans les interactions au sein des écosystèmes. Il est par ailleurs difficile de produire une connaissance sur l'évolution de cette érosion : les espèces comme les écosystèmes sont capables d'innovation, de création et de résilience. En cela, la perte du vivant est plus difficilement saisissable par les pouvoirs publics. La culture politique ne saurait centrer son action sur un phénomène qu'elle ne parvient pas à s'approprier avec les instruments qui sont à sa disposition. Même si l'approche fixiste par espèces actuelle présente l'avantage pour l'action publique de pouvoir appréhender clairement le vivant, elle occulte la complexité du système et sa perte, que personne ne peut véritablement chiffrer et comptabiliser. C'est en ce sens que la perte globale de biodiversité reste en l'état actuel une aporie politique : elle représente un problème à peine percevable et qui ne peut donc être pris en charge par le politique.

\section{Remerciements}

Les auteurs tiennent à remercier Bruno Villalba pour sa relecture et ses précieux conseils. 


\section{Bibliographie}

Ameisen J.-C., Les chants mêlés de la Terre et de l'Humanité. Dialogues avec N. Truong, Paris, Le Monde/L'Aube, 2015.

Alphandéry P., Fortier A., 201 1, Les associations dans le processus de rationalisation des données naturalistes, Natures Sciences Sociétés, no 19, vol. 1, p. 22-30.

Alphandéry P., Fortier A., Sourdril A., 2012, Les données entre normalisation et territoire : la construction de la trame verte et bleue, Développement durable et territoires, $\mathrm{n}^{\circ} 2$, vol. 3, consulté le 30 septembre 2016. [http://developpementdurable.revues.org/9282].

Alphandéry P., Fortier A., 2013, Les données sur la nature entre rationalisation et passion, Revue du MAUSS, $\mathrm{n}^{\circ} 42$, vol. 2, p. 202-220.

Alphandéry P., Fortier A., 2015, Les données naturalistes à l'épreuve de la transparence, Études rurales, nº 195, vol. 1, p. 127-144.

Arpin I., Charvolin F., Fortier A., 2015, Les inventaires naturalistes : des pratiques aux modes de gouvernement. Introduction, Études rurales, no 195, vol. 1, p. 11-26.

Bezes P., 2012, État, experts et savoirs managériaux. Les producteurs et diffuseurs du New Public Management en France depuis les années 1970, Actes de la recherche en sciences sociales, $\mathrm{n}^{\circ} 193$, vol. 3, p. 16-37.

Blandin P., 2009, De la protection de la nature au pilotage de la biodiversité, Versailles, Quae, 124 p.

Boisvert V., Vivien F.-D., 1998, Un prix pour la biodiversité : l'évaluation économique entre différentes légitimités, Natures Sciences Sociétés, n ${ }^{\circ}$ 6, vol. 2, p. 17-26.

Bruno I., 2008, À vos marques, prêts... cherchez!, Bellecombeen-Bauges, Éditions du Croquant, 267 p.

Bruno I., Didier E., 2013, Benchmarking, l'État sous pression statistique, Zones, Paris, 209 p.

Charvolin F., 2003, L'invention de l'environnement en France, chroniques anthropologiques d'une institutionnalisation, Paris, La Découverte, 133 p.

Casetta E., Debord J., 2014, La biodiversité en question : enjeux philosophiques, éthiques et scientifiques, Paris, Éditions Matériologiques, $271 \mathrm{p}$.

Casetta E., 2014, Évaluer et conserver la société face au problème des espèces, in Casetta E., Debord J., La biodiver- sité en question : enjeux philosophiques, éthiques et scientifiques, Paris, Éditions Matériologiques, p. 139-154.

Couvet D., Vandevelde C., 2014, Biodiversité ordinaire : des enjeux écologiques au consensus social, in CASETTA E., Debord J., La biodiversité en question : enjeux philosophiques, éthiques et scientifiques, Paris, Éditions Matériologiques, p. 181-208.

Dardot P., Laval C., 2009, La nouvelle raison du monde. Essai sur la société néolibérale, Paris, La Découverte, 497 p.

Darwin C., 2009, 1862, De l'origine des espèces, Paris, Champion, $918 \mathrm{p}$.

Desrosières A., 1993, La politique des grands nombres, Paris, La Découverte, 437 p.

Granjou C., 2015, Sociologie des changements environnementaux. Pour un futur de la nature, Londres, ISTE, $189 \mathrm{p}$.

Henry O., Pierru F., 2012, Les consultants et la réforme des services publics, Actes de la recherche en sciences sociales, $\mathrm{n}^{\circ} 193$, p. 4-15.

Hoquet T., 2014, Métaphysique et biologie, in Hoquet T. (dir.), Précis de philosophie de biologie, Paris, Vuibert, p. 305319.

Lascoumes P., Le Galès P., 2005, « L'action publique saisie par ses instruments », in Lascoumes P., Le Galès P. (dir.), Gouverner par les instruments, Paris, Presses de Sciences Po, coll. «Gouvernance », p. 11-44.

Ministère de l'Écologie et du Développement durable, 2004, Stratégie Française pour la biodiversité. Enjeux, finalités, orientations, Paris, 48 p.

Ogien A., 2010, La valeur sociale du chiffre, Revue française de socio-économie, $\mathrm{n}^{\circ}$ 5, vol. 1, p. 19-40.

Perret B., 2008, L'évaluation des politiques publiques, Paris, La Découverte, $120 \mathrm{p}$.

Pierru F., 2007, Hippocrate malade de ses réformes, Bellecombeen-Bauges, Éditions du Croquant, 319 p.

Porter T.M., 1995, Trust in numbers. The pursuit of objectivity in science and public life, Princeton, Princeton University Press, $310 \mathrm{p}$.

Prochiantz A., 2012, Qu'est-ce que le vivant?, Paris, Seuil, $171 \mathrm{p}$.

SALAis R., 2010, Usages et mésusages de l'argument statistique : le pilotage des politiques publiques par la performance, Revue française des affaires sociales, n 1, p. 129-147. 